PACS Numbers: 73.40.Gk, 78.20.Bh, 78.66.-w, 85.30.Vw

\title{
Resonant electron transfer between quantum dots
}

\author{
Leonid A. Openov \\ Moscow State Engineering Physics Institute (Technical University) \\ 115409 Moscow, Russia \\ E-mail: opn@supercon.mephi.ru
}

\begin{abstract}
An interaction of electromagnetic field with a nanostructure composed of two quantum dots is studied theoretically. An effect of a resonant electron transfer between the localized low-lying states of quantum dots is predicted. A necessary condition for such an effect is the existence of an excited bound state whose energy lies close to the top of the barrier separating the quantum dots. This effect may be used to realize the reversible quantum logic gate NOT if the superposition of electron states in different quantum dots is viewed as the superposition of bits 0 and 1 .
\end{abstract}

\section{INTRODUCTION}

One way to overcome the limitations of present semiconductor microelectronics is to reduce the dimensions of electronic devices well below $100 \mathrm{~nm}$ size range. Novel device concepts are based on the use of quantum effects in nanostructures [1]. While a great number of technological problems still remains to be resolved, there is a considerable experimental and theoretical activity in the field. Among other things, an interaction of electromagnetic field with nanostructures is of particular interest since it results in a variety of phenomena highlighting the wave nature of electrons (see, e.g., [2]).

Grossmann et al. 30 have shown that a laser with appropriate power and frequency can force the electron in a double-well nanostructure to stay in one of the wells. In this paper we draw attention to a possibility of an opposite effect, a laser-induced electron transfer between two quantum dots situated so far from each other that an electron placed initially in one of the dots may be thought of as localized in that dot, while having been transferred to the other dot, the electron remains localized in it after the laser pulse is off.

The paper is organized as follows. We begin with qualitative estimates of characteristic energies and times of a double-dot nanostructure and description of an appropriate model. Next we study the temporal evolution of an electron under the influence of a classical electromagnetic field making use of the resonant approximation. We demonstrate that the frequency, amplitude and duration of an electromagnetic pulse may be adjusted in such a way that an electron will be transferred from the localized lowest-energy state of one of the quantum dots to the localized lowest-energy state of another quantum dot with a probability close to unity. We discuss a possibility of using this effect to realize the quantum logic gate NOT.

\section{QUALITATIVE ESTIMATES}

We consider two semiconducting quantum dots, $A$ and $B$, such that each quantum dot, when isolated, has two size-quantized energy levels in the conduction band. Let us denote the energies of the lower level $|\alpha 1\rangle$ and the upper level $|\alpha 2\rangle$ by $\varepsilon_{\alpha 1}$ and $\varepsilon_{\alpha 2}$ respectively, where $\alpha=A$ or $B$ is the dot index, and the energies are measured from the bottom of the conduction band. For the sake of simplicity we assume the quantum dots to be identical, i.e., the values of $\varepsilon_{\alpha 1} \equiv \varepsilon_{1}$ and $\varepsilon_{\alpha 2} \equiv \varepsilon_{2}$ do not depend on $\alpha\left(\varepsilon_{2}>\varepsilon_{1}\right)$. The wave functions $\langle\mathbf{r} \mid \alpha 1\rangle$ for the dots $\alpha=A$ and $B$ have the same functional form but are centered in different regions of coordinate space, this is also true for the wave functions $\langle\mathbf{r} \mid \alpha 2\rangle$ of the excited states.

If the distance $d$ between the quantum dots and the height $U$ of the energy barrier separating the dots are reasonably large, the wave functions $\langle\mathbf{r} \mid \alpha 1\rangle$ for $\alpha=A$ and $B$ are strongly localized in the vicinity of the corresponding quantum dot within the region of the $\operatorname{dot}$ size $a$. Hence, their overlap can be neglected. In other words, the state $|\alpha 1\rangle$ with the energy $\varepsilon_{1}$ may be thought of as doubly degenerate with respect to the dot index $\alpha$, i.e., with respect to electron location, either in the $\operatorname{dot} A$ or in the $\operatorname{dot} B$.

It should be stressed that an electron may be considered as localized in one of the dots in the state $|\alpha i\rangle(i=1$ or 2 specifies the energy level) if we are interested in the processes whose characteristic times are much shorter than the time $\tau_{i}$ it takes for an electron to turn between the states $|A i\rangle$ and $|B i\rangle$. The value of $\tau_{i}$ may be estimated as

$$
\tau_{i} \approx \hbar / V_{i}
$$


where $V_{i}$ is the energy of electron hopping between the states $|A i\rangle$ and $|B i\rangle$. According to Landau and Lifshitz 雊, in the quasi-classical approximation one has

$$
V_{i} \approx \frac{\hbar}{T_{i}} \exp \left(-\frac{d}{\hbar} \sqrt{2 m^{*}\left(U-\varepsilon_{i}\right)}\right)
$$

where $T_{i}=\sqrt{2 m^{*} a^{2} / \varepsilon_{i}}$ is a period of a classical motion for an electron with the energy $\varepsilon_{i}$ in the quantum dot, and $m^{*}$ is the electron effective mass (for the sake of simplicity, we assume the values of $m^{*}$ to be the same in the dots and in the barrier). For the quantum dot cubic in shape, the ground state energy $\varepsilon_{1}$ may be estimated as $\varepsilon_{1} \approx 3 \pi^{2} \hbar^{2} / 2 m^{*} a^{2}$ provided that $\varepsilon_{1}<<U$. Then $T_{i} \approx \hbar \pi \sqrt{3 / \varepsilon_{1} \varepsilon_{i}}$, and one has from (2):

$$
V_{i} \approx \frac{1}{\gamma} \sqrt{\varepsilon_{1} \varepsilon_{i}} \exp \left(-\gamma \frac{d}{a} \sqrt{\frac{U-\varepsilon_{i}}{\varepsilon_{1}}}\right),
$$

where $\gamma=\pi \sqrt{3}$ is a numerical coefficient.

Taking $U \approx 1 \mathrm{eV}, \varepsilon_{1} \approx 0.1 \mathrm{eV}$, and $d / a \approx 3$, we obtain $V_{1} \sim 10^{-23} \mathrm{eV}$. Hence, a characteristic time it takes for an electron to tunnel from the ground state of one dot to the ground state of another dot, $\tau_{1} \sim 10^{8} \mathrm{~s}$, is very long even on a macroscopic scale, and such a tunneling can be ignored. On the other hand, if the energy $\varepsilon_{2}$ of the excited bound state is close to $U$, then the value of $V_{2}$ is many orders of magnitude greater than $V_{1}$. Taking, e.g., $U-\varepsilon_{2} \approx 0.01 \mathrm{eV}$, we have $V_{2} \sim 10^{-3} \mathrm{eV}$ and $\tau_{2} \sim 10^{-12} \mathrm{~s}$. Thus, for a certain set of quantum dots parameters, the low-lying energy level of the dots can be viewed as degenerate, whereas the excited level splits into two sublevels with the energies $\varepsilon_{2} \pm V_{2}$. It is important for the following consideration that the electron wave functions of the resulting excited sublevels are not localized within a particular dot, but spread over both dots as $(\langle\mathbf{r} \mid A 2\rangle \pm\langle\mathbf{r} \mid B 2\rangle) / \sqrt{2}$.

Of course, our estimates of $V_{i}$ and $\tau_{i}$ are rather crude, they strongly depend on the supposed form of confinement potential and should be considered as qualitative. However, one can hope that, first, for a dot of an arbitrary shape it is possible to shift the energy of one of excited states very close to the continuum part of the energy spectrum by varying, e.g., the dot size and the doping level, and, second, the distance between two such quantum dots can be adjusted to satisfy the condition $V_{2}>>V_{1}$ for the energies of electron hopping between the excited states and between the ground states of the dots respectively (and hence, the condition $\tau_{2}<<\tau_{1}$ for the times of electron switching between those pairs of states).

It should be pointed out that except for the times $\tau_{1}$ and $\tau_{2}$, there is one more important time scale, the lifetime $\tau^{*}$ of electron in the excited state with respect to spontaneous transition to the ground state at the sacrifice of photon or phonon emission. It has been shown by Nomoto et al. [5] that the value of $\tau^{*}$ strongly depends on the dot size and can be as long as $10^{-6} \mathrm{~s}$ or even longer, so that one can suppose $\tau_{2}<<\tau^{*}<<\tau_{1}$.

From the above line of reasoning, we set $V_{1}=0$ (i.e., $\tau_{1}=\infty$ ). We denote $V_{2} \equiv V$. The diagram of one-electron energy levels is shown schematically in Fig. 1. The overall idea is to make use of one of the excited states of the system to induce electron transitions between the lowest-energy states localized in different quantum dots under the influence of resonant external perturbation (e.g., an ac electromagnetic field). According to the laws of quantum mechanics an electron, having been "raised up" (at some moment in time) by the perturbation from the localized state, e.g., $|A 1\rangle$, to the excited state, e.g., $(|A 2\rangle+|B 2\rangle) / \sqrt{2}$, immediately becomes spread over both dots, so that it can be subsequently "lowered down" by the same perturbation (acting on both dots) to the localized state of the other quantum dot, in our example $|B 1\rangle$. The physical picture of such an effect seems quite clear, the question is only in the probability of electron transfer between the dots.

As to the case of an isolated quantum dot, it is well known that a periodic perturbation $\hat{F} \cos (\Omega t)$ with frequency $\Omega=\epsilon_{2}-\epsilon_{1}$ (hereinafter $\hbar=1$ ) leads to periodic oscillations of the probabilities $p_{1}(t)$ and $p_{2}(t)$ of detecting an electron in levels $|1\rangle$ and $|2\rangle$ [4]. I]. If $p_{1}(0)=1$ and $p_{2}(0)=0$ at the initial moment, then

$$
p_{2}(t)=\sin ^{2}\left(\omega_{R} t\right)
$$

where $\omega_{R}=|\langle 2|\hat{F}| 1\rangle| / 2$. Here $\langle 2|\hat{F}| 1\rangle$ is the matrix element of the interlevel transition. It follows from Eq. (凿) that one can select the time $T$ during which the perturbation is on (for example, $T=\pi / 2 \omega_{R}$ ) so that the condition $p_{2}(T)=1$ is satisfied (so called $\pi$-pulse). Below we shall show that in the case of the double-dot system, the probability of electron transfer between the localized low-lying states of quantum dots can also be put very close to unity through the proper choice of the characteristics of an electromagnetic pulse. Similar effect in semiconducting quantum wells has been discussed in [6].

\section{DESCRIPTION OF THE MODEL}


Let the external perturbation be the classical ac electric field $\mathbf{E}(t)$. Then the model Hamiltonian has the form

$$
\hat{H}(t)=\epsilon_{1}\left(\hat{a}_{A 1}^{+} \hat{a}_{A 1}+\hat{a}_{B 1}^{+} \hat{a}_{B 1}\right)+\epsilon_{2}\left(\hat{a}_{A 2}^{+} \hat{a}_{A 2}+\hat{a}_{B 2}^{+} \hat{a}_{B 2}\right)-V\left(\hat{a}_{A 2}^{+} \hat{a}_{B 2}+\text { h.c. }\right)+\mathbf{E}(t)\left[\mathbf{d}\left(\hat{a}_{A 2}^{+} \hat{a}_{A 1}+\hat{a}_{B 2}^{+} \hat{a}_{B 1}\right)+\text { h.c. }\right],
$$

where $\hat{a}_{\alpha i}^{+}\left(\hat{a}_{\alpha i}\right)$ is the electron creation (annihilation) operator for an electron in states $|\alpha i\rangle(\alpha=A, B ; i=1,2) ; \mathbf{d}=$ $\langle A 2|-e \mathbf{r}| A 1\rangle=\langle B 2|-e \mathbf{r}| B 1\rangle$ is the matrix element of optical dipole transitions $|A 1\rangle \rightleftharpoons|A 2\rangle$ and $|B 1\rangle \rightleftharpoons|B 2\rangle$. We do not specify the spin index explicitly since we consider a single electron whose spin projection on an arbitrary chosen axis remains unchanged upon the action of ac electric field. Note that in Eq.(5) we have omitted the terms describing both tunnel and optical transitions $|A 1\rangle \rightleftharpoons|B 2\rangle$ and $|B 1\rangle \rightleftharpoons|A 2\rangle$ since the wave functions entering into the corresponding matrix elements are centered in different quantum dots (one of wave functions being strongly localized within a particular dot), and hence one can expect those matrix elements be exponentially smaller than $V$ and $d$ respectively.

Let us suppose that the external field is turned on at $t=0$ and turned off at $t=T$, and has a frequency $\Omega$, i.e.,

$$
\mathbf{E}(t)=\mathbf{E}_{\mathbf{0}} \cos (\Omega t) \theta(t) \theta(T-t),
$$

where $\mathbf{E}_{\mathbf{0}}$ is the field amplitude, $\theta(t)$ is the Heaviside step function. The pulse duration $T$ and frequency $\Omega$ are to be derived by maximizing the probability that an electron is transferred from the localized low-lying state of one quantum dot to that of another dot.

It is convenient to introduce new notations for one-electron states:

$$
|1\rangle=|A 1\rangle, \quad|2\rangle=|B 1\rangle, \quad|3\rangle=(|A 2\rangle+|B 2\rangle) / \sqrt{2}, \quad|4\rangle=(|A 2\rangle-|B 2\rangle) / \sqrt{2},
$$

and hence to replace in Eq.(5) the operators $\hat{a}_{A 1}$ and $\hat{a}_{B 1}$ by operators $\hat{a}_{1}$ and $\hat{a}_{2}$ respectively as well as to go from operators $\hat{a}_{A 2}$ and $\hat{a}_{B 2}$ describing the excited states of isolated quantum dots with the energy $\epsilon_{2}$ to operators $\hat{a}_{3}=\left(\hat{a}_{A 2}+\hat{a}_{B 2}\right) / \sqrt{2}$ and $\hat{a}_{4}=\left(\hat{a}_{A 2}-\hat{a}_{B 2}\right) / \sqrt{2}$ describing the excited sublevels of the double-dot nanostructure with energies $\epsilon_{2}-V$ and $\epsilon_{2}+V$ respectively. Then the time-independent part of the Hamiltonian acquires a diagonal form, while optical transitions take place between the states $|1\rangle \rightleftharpoons|3\rangle,|2\rangle \rightleftharpoons|3\rangle,|1\rangle \rightleftharpoons|4\rangle$, and $|2\rangle \rightleftharpoons|4\rangle$ :

$$
\hat{H}(t)=\epsilon_{1}\left(\hat{a}_{1}^{+} \hat{a}_{1}+\hat{a}_{2}^{+} \hat{a}_{2}\right)+\left(\epsilon_{2}-V\right) \hat{a}_{3}^{+} \hat{a}_{3}+\left(\epsilon_{2}+V\right) \hat{a}_{4}^{+} \hat{a}_{4}+\mathbf{E}(t)\left[\frac{\mathbf{d}}{\sqrt{2}}\left(\hat{a}_{3}^{+} \hat{a}_{1}+\hat{a}_{3}^{+} \hat{a}_{2}+\hat{a}_{4}^{+} \hat{a}_{1}-\hat{a}_{4}^{+} \hat{a}_{2}\right)+\text { h.c. }\right] .
$$

For the system under consideration, the one-electron wave function $\Psi(t)$ can be expressed at any moment as

$$
\Psi(t)=\sum_{i=1}^{4} A_{i}(t) \exp \left(-i E_{i} t\right)|i\rangle,
$$

where $E_{i}$ are eigenvalues of the stationary Schrödinger equation $\hat{H}|i\rangle=E_{i}|i\rangle$ in the absence of an applied field $(t \leq 0)$ :

$$
E_{1}=\epsilon_{1}, \quad E_{2}=\epsilon_{1}, \quad E_{3}=\epsilon_{2}-V, \quad E_{4}=\epsilon_{2}+V .
$$

The values $A_{i}(0)$ define the electron wave function at the initial moment; $A_{i}(0)=A_{i}(t<0)$ since $|i\rangle$ are eigenstates of the Hamiltonian (8) for $t \leq 0$. We assume that at $t \leq 0$ an electron is localized in the ground state of the $\operatorname{dot} A$, i.e., $A_{1}(0)=1, A_{2}(0)=A_{3}(0)=A_{4}(0)=0$. The probability $p_{i}(t)$ to find an electron in state $|i\rangle$ at an arbitrary time $t$ is $\left|A_{i}(t)\right|^{2}$ (it follows from the normalization condition that $p_{1}(t)+p_{2}(t)+p_{3}(t)+p_{4}(t)=1$ at any $t$ ). In particular, the value of $p_{2}(t)$ is the probability that an electron occupies the low-lying localized level of the dot $B$ at a time $t$. The coefficients $A_{i}(t)$ in Eq.(9) can be calculated by solving the time-dependent Schrödinger equation

$$
i \frac{\partial \Psi(t)}{\partial t}=\hat{H}(t) \Psi(t)
$$

where $\hat{H}(t)$ is given by Eq.(8), i.e., it explicitly depends on time at $0 \leq t \leq T$.

\section{RESONANT APPROXIMATION}

In order to solve the problem analytically, we use the resonant approximation [4. We assume that the frequency $\Omega$ of ac electric field is close to the resonant frequency

$$
\Omega_{r}=\epsilon_{2}-V-\epsilon_{1},
$$


i.e., to the difference between the energy $\epsilon_{2}-V$ of the lower excited state $|3\rangle$ and the energy $\epsilon_{1}$ of the two-fold degenerate ground state $|1\rangle(|2\rangle)$ so that those three states are resonant with the ac field, while the upper excited state $|4\rangle$ with the energy $\epsilon_{2}+V$ is out of resonance. To be precise, the value of $\Omega$ should be much more closer to $\epsilon_{2}-V-\epsilon_{1}$ than to $\epsilon_{2}+V-\epsilon_{1}$, i.e., the following strong inequality should be satisfied:

$$
|\delta|<<V,
$$

where the value of

$$
\delta=\Omega-\Omega_{r}
$$

quantifies the offset from resonance. Since in the resonant approximation the electron transitions $|1\rangle \rightleftharpoons|4\rangle$ and $|2\rangle \rightleftharpoons|4\rangle$ can be ignored, the effective Hamiltonian has the form

$$
\hat{H}(t)=\epsilon_{1}\left(\hat{a}_{1}^{+} \hat{a}_{1}+\hat{a}_{2}^{+} \hat{a}_{2}\right)+\left(\epsilon_{2}-V\right) \hat{a}_{3}^{+} \hat{a}_{3}+\left[\frac{\lambda}{2} \exp (-i \Omega t)\left(\hat{a}_{3}^{+} \hat{a}_{1}+\hat{a}_{3}^{+} \hat{a}_{2}\right)+\text { h.c. }\right],
$$

where we have introduced the notation

$$
\lambda=\mathbf{E}_{\mathbf{0}} \mathbf{d} / \sqrt{2} .
$$

Generally speaking, the Schrödinger equation (11) with Hamiltonian (15) can be reduced to the system of coupled differential equations for the coefficients $A_{i}(t)$ in the expansion (9) of the wave function $(i=1-3)$. It is more convenient, however, to go to a representation with a time-independent Hamiltonian making use of the unitary transformation

$$
\hat{U}(t)=\exp \left[\frac{i \Omega t}{2}\left(\hat{a}_{1}^{+} \hat{a}_{1}+\hat{a}_{2}^{+} \hat{a}_{2}-\hat{a}_{3}^{+} \hat{a}_{3}\right)\right]
$$

similar to those proposed by Galitskii et al. [8] to describe the interaction of a semiconductor with a strong electromagnetic field. Substituting

$$
\Psi(t)=\hat{U}(t) \tilde{\Psi}(t)
$$

into the Schrödinger equation (11) for $\Psi(t)$, we obtain the Schrödinger equation for $\tilde{\Psi}(t)$ :

$$
i \frac{\partial \tilde{\Psi}(t)}{\partial t}=\hat{\tilde{H}} \tilde{\Psi}(t)
$$

with the Hamiltonian

$$
\begin{aligned}
\hat{\tilde{H}}= & \hat{U}^{+}(t) \hat{H}(t) \hat{U}(t)-i \hat{U}^{+}(t) \frac{\partial \hat{U}(t)}{\partial t} \\
& =\left(\epsilon_{1}+\Omega / 2\right)\left(\hat{a}_{1}^{+} \hat{a}_{1}+\hat{a}_{2}^{+} \hat{a}_{2}\right)+\left(\epsilon_{2}-V-\Omega / 2\right) \hat{a}_{3}^{+} \hat{a}_{3}+\left[\frac{\lambda}{2}\left(\hat{a}_{3}^{+} \hat{a}_{1}+\hat{a}_{3}^{+} \hat{a}_{2}\right)+\text { h.c. }\right] .
\end{aligned}
$$

Since the Hamiltonian $\hat{\tilde{H}}$ is independent on time, the general solution of the time-dependent Schrödinger equation (19) for $0 \leq t \leq T$ has the form:

$$
\tilde{\Psi}(t)=\sum_{i=1}^{3} B_{i} \exp \left(-i \tilde{E}_{i} t\right)|\tilde{i}\rangle,
$$

where $|\tilde{i}\rangle$ and $\tilde{E}_{i}$ are the eigenstates and eigenvalues of the stationary Schrödinger equation

$$
\hat{\tilde{H}}|\tilde{i}\rangle=\tilde{E}_{i}|\tilde{i}\rangle .
$$

We seek solutions of Eq. (22) in the form

$$
|\tilde{i}\rangle=\sum_{k=1}^{3} C_{i k}|k\rangle
$$


where $|k\rangle$ are eigenstates defined by Eq.(7) for $t \leq 0$. Substituting Eq.(23) into Eq.(22), we obtain a set of equations for $C_{i k}$ and $\tilde{E}_{i}$ :

$$
\sum_{k=1}^{3} C_{i k}\left(\langle i|\hat{\tilde{H}}| k\rangle-\delta_{i k} \tilde{E}_{i}\right)=0,
$$

where $i=1 \div 3$, and $\langle i|\hat{\tilde{H}}| k\rangle$ are the matrix elements of the Hamiltonian (20) in terms of the first three basis states of (7). The Hamiltonian matrix $\langle i|\hat{\tilde{H}}| k\rangle$ has the form

$$
\left(\begin{array}{ccc}
\epsilon_{1}+\Omega / 2 & 0 & \lambda^{*} / 2 \\
0 & \epsilon_{1}+\Omega / 2 & \lambda^{*} / 2 \\
\lambda / 2 & \lambda / 2 & \epsilon_{2}-V-\Omega / 2
\end{array}\right) .
$$

From Eqs. (21) and (23) one has

$$
\tilde{\Psi}(t)=\sum_{i=1}^{3} D_{i}(t)|i\rangle
$$

where

$$
D_{i}(t)=\sum_{k=1}^{3} B_{k} C_{k i} \exp \left(-i \tilde{E}_{k} t\right) .
$$

Since $\tilde{\Psi}(0)=\Psi(0)$, see Eqs. (17) and (18), we have $D_{i}(0)=A_{i}(0)$, where the coefficients $A_{i}(0)$ determine the state (9) for $t \leq 0$. Therefore, from Eq.(27) we obtain the equations that determine the coefficients $B_{i}$ in terms of given $A_{i}(0)$ :

$$
A_{i}(0)=\sum_{k=1}^{3} B_{k} C_{k i}
$$

whence

$$
B_{i}=\sum_{k=1}^{3} A_{k}(0) C_{k i}^{-1}
$$

where $C^{-1}$ is the matrix inverse of $C$. From Eqs. (27) and (29) we obtain :

$$
D_{i}(t)=\sum_{k=1}^{3} \sum_{l=1}^{3} A_{l}(0) C_{l i}^{-1} C_{k i} \exp \left(-i \tilde{E}_{k} t\right) .
$$

Finally, given Eq. 18 relating the function $\tilde{\Psi}(t)$ to $\Psi(t)$ and taking into account that the operator $\hat{U}(t)$ defined by Eq.(17) is unitary, we obtain an expression for the probability $p_{i}(t)$ for the transition to the state $|i\rangle$ :

$$
p_{i}(t)=\left|D_{i}(t)\right|^{2} .
$$

\section{RESULTS AND DISCUSSION}

Since we assume (see above) that at $t \leq 0$ an electron is localized in the level $|1\rangle$, the lowest energy level of the $\operatorname{dot} A$, i.e, $A_{1}(0)=1$ and $A_{2}(0)=A_{3}(0)=0$, expressions (30) and (31) simplify somewhat. Having calculated the eigenvalues $\tilde{E}_{i}$ and the matrix of eigenvectors $C_{i k}$ from Eq.(24), we obtain from Eqs. (30) and (31) the expressions for the probabilities of transitions from the state $\Psi(0)=|1\rangle$ to the state $|i\rangle$ :

$$
\begin{aligned}
& p_{1}(t)=\cos ^{4}\left(\omega_{R} t\right)-\sin ^{2}\left(\frac{\delta t}{4}\right) \cos \left(2 \omega_{R} t\right)+\frac{\delta^{2}}{64 \omega_{R}^{2}} \sin ^{2}\left(2 \omega_{R} t\right)+\frac{\delta}{8 \omega_{R}} \sin \left(\frac{\delta t}{2}\right) \sin \left(2 \omega_{R} t\right), \\
& p_{2}(t)=\sin ^{4}\left(\omega_{R} t\right)+\sin ^{2}\left(\frac{\delta t}{4}\right) \cos \left(2 \omega_{R} t\right)+\frac{\delta^{2}}{64 \omega_{R}^{2}} \sin ^{2}\left(2 \omega_{R} t\right)-\frac{\delta}{8 \omega_{R}} \sin \left(\frac{\delta t}{2}\right) \sin \left(2 \omega_{R} t\right), \\
& p_{3}(t)=\frac{1}{2}\left(1-\frac{\delta^{2}}{16 \omega_{R}}\right) \sin ^{2}\left(2 \omega_{R} t\right),
\end{aligned}
$$


where $\delta$ is defined by Eqs. (12) and (14), and

$$
\omega_{R}=\frac{\sqrt{\delta^{2}+2|\lambda|^{2}}}{4} .
$$

In a particular case of exact resonance $(\delta=0)$, one has from Eq.(32):

$$
\begin{aligned}
& p_{1}(t)=\cos ^{4}\left(\omega_{R} t\right), \\
& p_{2}(t)=\sin ^{4}\left(\omega_{R} t\right), \\
& p_{3}(t)=\frac{1}{2} \sin ^{2}\left(2 \omega_{R} t\right) .
\end{aligned}
$$

We are interested mainly in the probability $p_{2}(t)$ of electron transfer to the level $|2\rangle$, the lowest energy level of the $\operatorname{dot} B$. It follows from Eq.(34) that $p_{2}(t)=1$ at $t=T_{n}$, where

$$
T_{n}=\frac{\pi}{2 \omega_{R}}+\frac{\pi n}{\omega_{R}},
$$

and $n$ is an integer. Hence, after the applied field is off at $t=T_{n}$, the electron will stay in the state $|2\rangle$ since this state is the eigenstate of the Hamiltonian (8) in the absence of external perturbation. So, if a characteristic time of electron tunneling between the states $|1\rangle$ and $|2\rangle$, the ground states of the dots $A$ and $B$ respectively, is long enough (i.e., if the two dots are placed far apart from each other and/or are separated by relatively high energy barrier), the electron remains, in fact, localized in the $\operatorname{dot} B$.

When the frequency is offset from resonance $(\delta \neq 0)$, the value of $p_{2}\left(T_{n}\right)$ derived from Eq.(32) deviates from unity by a quantity of order $\delta^{2} T_{n}^{2}$. At a given value of $\delta$, the probability $p_{2}\left(T_{n}\right)$ has a maximum for $n=0$, see Eq.(35), i.e., for the perturbation duration time $T_{0}=\pi / 2 \omega_{R}$ :

$$
p_{2}\left(T_{0}\right)=1-\frac{\pi^{2}}{64} \frac{\delta^{2}}{\omega_{R}^{2}} .
$$

Taking Eq.(33) into account, we are led to the following inequality

$$
|\delta|<<|\lambda|
$$

which should hold in order that the probability $p_{2}\left(T_{0}\right)$, Eq.(36), be very close to unity.

Note that the perturbing ac field acting for a finite period of time, $T_{0}$, contains harmonics in the frequency range $\delta \omega \approx 1 / T_{0}$. In order that the approximating Hamiltonian, Eq.(15), be valid, the bandwidth $\delta \omega$ should be much smaller than the interval $2 \mathrm{~V}$ between the energies of excited states $|3\rangle$ and $|4\rangle$ since otherwise the external field will mix all the states $|1\rangle,|2\rangle,|3\rangle$, and $|4\rangle$, see Eq.(17). Besides, the time $T_{0}$ needed to transfer an electron from the ground state $|1\rangle$ of the $\operatorname{dot} A$ to the ground state $|2\rangle$ of the $\operatorname{dot} B$ should be much shorter than the lifetime $\tau^{*}$ of electron in the "auxiliary" excited state $|3\rangle$ of the nanostructure, see Sec.2, since otherwise the probability of photon/phonon emission at $t \ll T_{0}$ is high, resulting in decoherence and breakdown of unitary electron evolution under the influence of ac field. Hence, taking into account that $T_{0} \sim 1 /|\lambda|$, see Eqs. (33), (35), and (37), we arrive at the following inequalities:

$$
\frac{1}{\tau^{*}}<<|\lambda|<<V .
$$

Finally, combining Eqs. (13), (37), and (38) together, one has the following conditions for $(i)$ applicability of the resonant approximation to the description of electronic transitions in the nanostructure under consideration and (ii) proximity of the probability of electron transfer from one dot to another to unity:

$$
\frac{1}{\tau^{*}}, \delta<<|\lambda|<<V .
$$

We note that conditions (39) imposed on the frequency, duration and amplitude of electromagnetic pulse can be fulfilled in the experiment. Indeed, if $V \sim 10^{-3} \mathrm{eV}$ (see estimates in Sec.2), we should have, e.g., $|\lambda| \approx 10^{-5}-10^{-4}$ $\mathrm{eV}$ and $|\delta| \approx 10^{-7}-10^{-6} \mathrm{eV}$ (our numerical calculations have shown that at $|\lambda| / V=0.1$ and $|\delta| /|\lambda|=0.1$ the value of $p_{2}\left(T_{0}\right)$ is about 0.99$)$. Since the parameter $|\lambda|$ is of the order of a product of the electric field amplitude $E_{0}$ and the optical dipole matrix element $d$, see Eq.(16), and $d \approx e a$, for a characteristic quantum dot size $a \approx 10 \mathrm{~nm}$ we 
obtain $E_{0} \approx 10-100 \mathrm{~V} / \mathrm{cm}$, which can be easily realized experimentally. As to the condition imposed on $\delta$, for assumed value of $V$ one finds that the frequency $\Omega$ of the external source should be accurate to within $10^{9} \mathrm{~s}^{-1}$ or better. Such an accuracy can be obtained by modern experimental methods. Besides, the resonance condition can probably be achieved by varying the energy difference between the size-quantized levels via applying a gate voltage to the nanostructure.

From symmetry considerations it is clear that if at $t \leq 0$ an electron is in the state $|2\rangle$ (i.e. in the lowest-energy state of the $\operatorname{dot} B$ ), it will take the same time $T_{0}$ to transfer it to the state $|1\rangle$ (i.e. to the lowest-energy state of the $\operatorname{dot} A)$ as the time needed for electron transfer from the state $|1\rangle$ to the state $|2\rangle$, see above. Hence, if initially an electron is in an arbitrary superposition of states $|1\rangle$ and $|2\rangle$, i.e. $\Psi(0)=\alpha|1\rangle+\beta|2\rangle$ where $\alpha$ and $\beta$ are complex numbers such that $|\alpha|^{2}+|\beta|^{2}=1$, then $\Psi\left(T_{0}\right)=\beta|1\rangle+\alpha|2\rangle$.

Now it is in order to mention a possible application of the effect of ac field-induced electron transfer between two quantum dots to the so called "quantum computation" [9.10]. Indeed, if the states $|1\rangle$ and $|2\rangle$, i.e. electron locations in dots $A$ and $B$, are viewed as the Boolean states 0 and 1 respectively, then their linear combination $\alpha|1\rangle+\beta|2\rangle$ may be viewed as a "quantum bit" ("qubit"). In its turn, an action of the resonant (in the sense discussed above) ac field on the double-dot nanostructure may be considered as a unitary operation NOT over the qubit:

$$
U_{N O T}\left(\begin{array}{c}
\alpha \\
\beta
\end{array}\right)=\left(\begin{array}{c}
\beta \\
\alpha
\end{array}\right) \text {. }
$$

Such an operation is non-dissipative (reversible). Hence, the double-dot nanostructure can function as a reversible logic gate NOT (inverter), in contrast to a number of dissipative (non-reversible) logic circuits proposed in the literature (see, e.g., [5] 11 13]).

Various schemes for realizing reversible quantum logic gates have also been discussed (see, e.g., 9, 14 17]) and demonstrated experimentally [18 20]. Almost all of these schemes are based on encoding qubits in either photon states or in nuclear spins. From the perspective of high density computational circuits, the reversible logic gates based on single electrons in quantum dots seem to be very appealing. Several quantum gate mechanisms based on electron spins in strongly coupled adjacent quantum dots have been proposed (see, e.g., 21 24]). In our opinion, encoding qubits in electron locations (i.e., in fact, in ground states of weakly coupled well separated quantum dots) rather than in electron spins may have an advantage that such qubits are well defined and can be expected to have long dephasing times. Besides, the measurement procedure may appear to be more straightforward than in the case of spin-based qubits.

Of course, a quantum inverter is the simplest logic gate. It operates with one qubit only and is not a universal gate, i.e. it cannot be considered as the fundamental building block of a quantum computer. However the ideas presented in this paper can be used to construct more complex gates consisting of several quantum dots and operating with more than one qubit, e.g. XOR (controlled-NOT) gate.

In conclusion, we have shown that a resonant electron transfer between the states localized in distant quantum dots can take place upon the influence of a resonant ac field with properly chosen characteristics (amplitude, frequency, and duration). Such a transfer occurs via an excited bound state of the double-dot nanostructure delocalized over both dots. Although only the simplest case that each dot has two size-quantized energy levels has ben considered, it is clear that, in general, the resonant electron transfer between the dots can be assisted by any excited level whose energy lies close to the top of the barrier separating the dots if the appropriate resonance conditions are satisfied. Since an electron can be in a quantum-mechanical superposition of states localized in different dots, the double-dot nanostructure can play a role of a reversible logic gate NOT operating the quantum bits.

\section{ACKNOWLEDGMENTS}

This work was supported by the Russian Federal Program "Integration", the Russian Foundation for Fundamental Research under Grant No 96-02-18918 and by the Russian State Program "Advanced Technologies and Devices in Micro- and Nanoelectronics". The author would like to thank V. V. V'yurkov, V. F. Elesin, and S. N. Molotkov for useful discussions. 
[1] H. Lüth, Phys. Stat. Sol. (b) 192, 287 (1995).

[2] A. V. Krasheninnikov, S. N. Molotkov, S. S. Nazin, and L. A. Openov, Zh. Eksp. Teor. Fiz. 112, 1257 (1997) [JETP 85, $682(1997)]$.

[3] F. Grossmann, T. Dittrich, P. Jung, and P.Hänggi, Phys. Rev. Lett. 67, 516 (1991).

[4] L. D. Landau and E. M. Lifshitz, 3rd ed., Nauka, Moscow (1974) [Pergamon Press, New York (1977)].

[5] K. Nomoto, R. Ugajin, T. Suzuki, and I. Hase, J. Appl. Phys. 79, 291 (1996).

[6] A. A. Gorbatsevich, V. V. Kapaev, and Yu. V. Kopaev, Zh. Eksp. Teor. Fiz. 107, 1320 (1995) [JETP 80, 734 (1995)].

[7] S. Flügge, Practical Quantum Mechanics II, Springer-Verlag, Berlin-Heidelberg-New York (1971).

[8] V. M. Galitskii, S. P. Goreslavskii, and V. F. Elesin, Zh. Eksp. Teor. Fiz. 57, 207 (1969) [JETP 30, 117 (1970)].

[9] S. Lloyd, Science 261, 1569 (1993).

[10] C. H. Bennett, Physics Today, October 1995, p.24.

[11] S. Bandyopadhyay, V. P. Roychowdhury, and X. Wang, Phys. Low-Dim. Struct. 8/9, 28 (1995).

[12] S. N. Molotkov and S. S. Nazin, Pis'ma v ZhETF 62, 256 (1995) [JETP Lett. 62, 273 (1995)]; ZhETF 110, 1439 (1996) [JETP 83, 794 (1996)].

[13] A. V. Krasheninnikov and L. A. Openov, Pis'ma v ZhETF 64, 214 (1996) [JETP Lett. 64, 231 (1996)].

[14] D. P. DiVincenzo, Science 270, 255 (1995).

[15] J. I. Cirac and P. Zoller, Phys. Rev. Lett. 74, 4091 (1995).

[16] B. E. Kane, Nature 393, 133 (1998).

[17] D. P. DiVincenzo, Nature 393, 113 (1998).

[18] Q. A. Turchette, C. J. Hood, W. Lange, H. Mabuchi, and H. J. Kimble, Phys. Rev. Lett. 75, 4710 (1995).

[19] C. Monroe, D. M. Meekhof, B. E. King, W. M. Itano, and D. J. Wineland, Phys. Rev. Lett. 75, 4714 (1995).

[20] I. L. Chuang, L. M. K. Vandersypen, X. Zhou, D. W. Leung, and S. Lloyd, Nature 393, 143 (1998).

[21] S. Bandyopadhyay and V. P. Roychowdhury, Superlattices and Microstructures 22, 411 (1997).

[22] A. M. Bychkov, L. A. Openov, and I. A. Semenihin, Pis'ma v ZhETF 66, 275 (1997) [JETP Lett. 66, 298 (1997)].

[23] D. Loss and D. P. DiVincenzo, Phys. Rev. A 57, 120 (1998).

[24] G. Burkard, D. Loss, and D. P. DiVincenzo, Phys. Rev. B 59, 2070 (1999). 


\section{FIGURE CAPTIONS}

Fig.1. Energy levels diagram of a nanostructure composed of two identical quantum dots, $A$ and $B$, see text for details. 


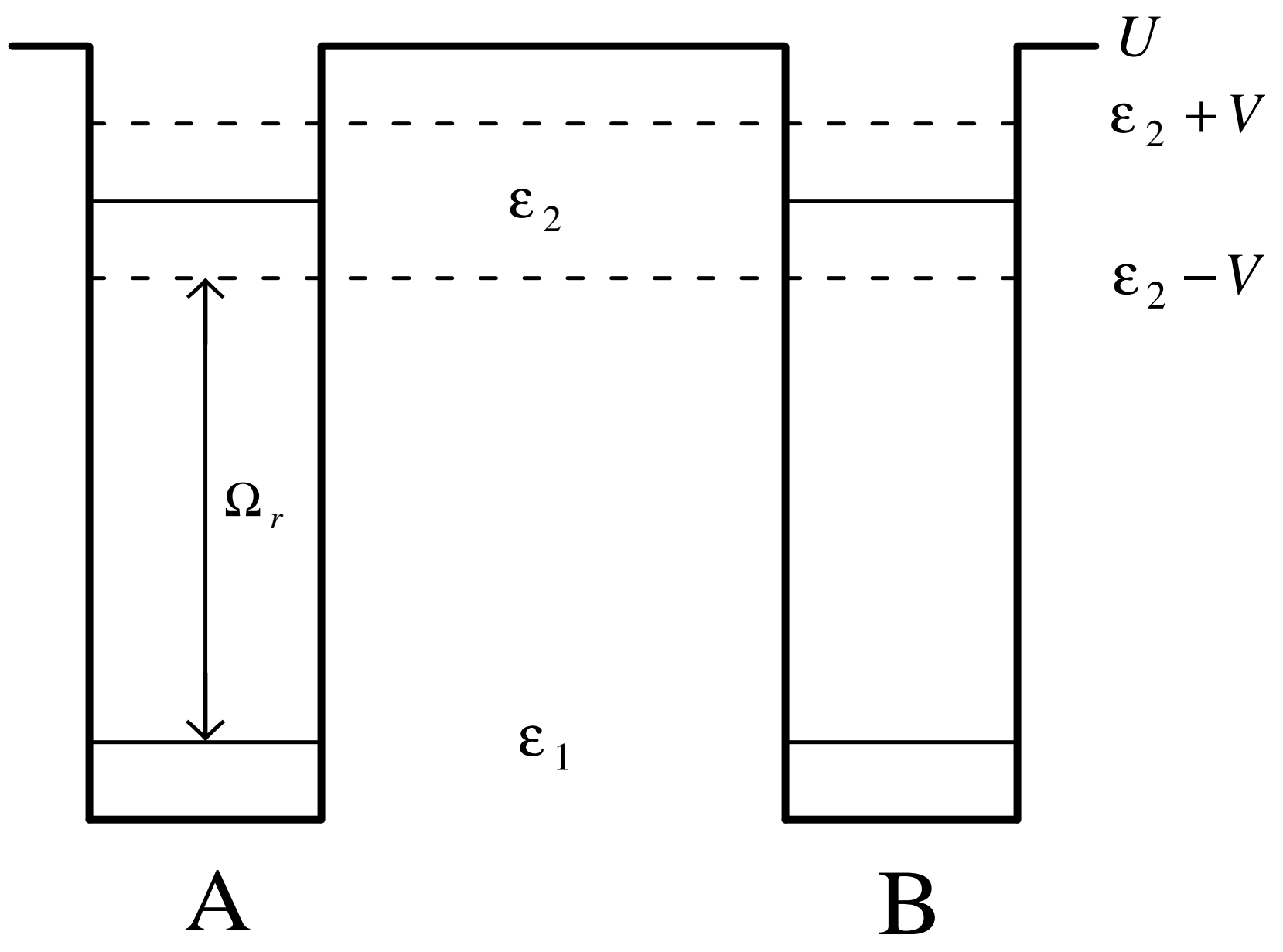

Figure 1 